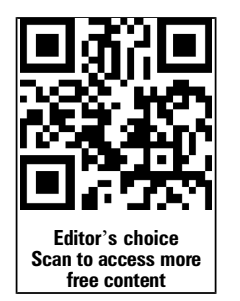

- Additional material is published online only. To view please visit the journal online (http://dx.doi.org/10.1136/ jclinpath-2014-202761).

${ }^{1}$ Department of Public Health, University of Naples Federico II, Naples, Italy

${ }^{2}$ Department of Clinical Medicine and Surgery, University of Naples Federico II, Napoles, Italy

${ }^{3}$ Department of Medicine (DIMES)_Anatomic Pathology Unit, Bellaria Hospital, University of Bologna, Bologna, Italy

${ }^{4}$ CNR/IEOS, Institute of Experimental Endocrinology and Oncology, National Research Council, Naples, Italy ${ }^{5}$ Department of Molecular Medicine and Medical Biotechnology (DMMBM)، University of Naples Federico II, Naples, Italy

\section{Correspondence to} Professor Giancarlo Troncone, Department of Public Health, University of Naples Federico II, via Sergio Pansini 5, Naples I-80131, Italy;

giancarlo.troncone@unina.it

Received 8 November 2014 Accepted 23 December 2014 Published Online First 21 January 2015

\title{
KRAS Mutant Allele-Specific Imbalance (MASI) assessment in routine samples of patients with metastatic colorectal cancer
}

\author{
Umberto Malapelle, ${ }^{1}$ Roberta Sgariglia, ${ }^{1}$ Alfonso De Stefano, ${ }^{2}$ Claudio Bellevicine, ${ }^{1}$ \\ Elena Vigliar, ${ }^{1}$ Dario de Biase, ${ }^{3}$ Romina Sepe, ${ }^{4,5}$ Pierlorenzo Pallante, ${ }^{4,5}$ \\ Chiara Carlomagno, ${ }^{2}$ Giovanni Tallini, ${ }^{3}$ Giancarlo Troncone ${ }^{1}$
}

\begin{abstract}
Aims Patients with colorectal cancer harbouring KRAS mutations do not respond to antiepidermal growth factor receptor (anti-EGFR) therapy. Community screening for KRAS mutation selects patients for treatment. When a KRAS mutation is identified by direct sequencing, mutant and wild type alleles are seen on the sequencing electropherograms. KRAS mutant allele-specific imbalance (MASI) occurs when the mutant allele peak is higher than the wild type one. The aims of this study were to verify the rate and tissue distribution of KRAS MASI as well as its clinical relevance.

Methods A total of 437 sequencing electropherograms showing KRAS exon 2 mutation was reviewed and in 30 cases next generation sequencing (NGS) was also carried out. Five primary tumours were extensively laser capture microdissected to investigated KRAS MASI tissue spatial distribution. KRAS MASI influence on the overall survival was evaluated in 58 patients. In vitro response to antiEGFR therapy in relation to different G13D KRAS MASI status was also evaluated.
\end{abstract}

Results On the overall, KRAS MASI occurred in 58/436 cases $(12.8 \%)$, being more frequently associated with G13D mutation ( $p=0.05)$ and having a heterogeneous tissue distribution. KRAS MASI detection by Sanger Sequencing and NGS showed 94\% (28/30) concordance. The longer overall survival of KRAS MASI negative patients did not reach statistical significance ( $p=0.08)$. In cell line model G13D KRAS MASI conferred resistance to cetuximab treatment.

Conclusions KRAS MASI is a significant event in colorectal cancer, specifically associated with G13D mutation, and featuring a heterogeneous spatial distribution, that may have a role to predict the response to EGFR inhibitors. The foreseen implementation of NGS in community KRAS testing may help to define KRAS MASI prognostic and predictive significance.

\section{INTRODUCTION}

Worldwide, a million patients are diagnosed with colorectal cancer (CRC) annually, and 50\% of them will one day develop metastatic disease. ${ }^{1}$ CrossMark Epidermal growth factor receptor (EGFR) is targeted by cetuximab and panitumumab monoclonal antibodies. $^{2}$ However, several clinical trials have demonstrated that approximately $40 \%$ patients, whose tumour has KRAS mutations, do not benefit from these drugs. ${ }^{3-6}$ Thus, community testing for KRAS mutation has been introduced and many laboratories adopt direct sequencing. When a KRAS mutation is identified by direct sequencing, mutant and wild type alleles are seen on the sequencing electropherograms. ${ }^{7}$ In some instances, even if the tumour has not been microdissected, the mutant allele appears to be in great excess of the wild type allele. ${ }^{8}$ The mutant allele may become dominant when deletion of the wild type allele and/or chromosome 12 hyperploidy or KRAS amplification occurs, leading to mutant allele-specific imbalance (MASI). ${ }^{8}$ This latter, in turn, leads to elevated KRAS mRNA level and increased GTPase activity. ${ }^{8}$ To date, little investigation has been dedicated to KRAS MASI. ${ }^{9-11}$ Only Hartman et al reported that in CRCs MASI occurs in $19.7 \%$ of KRAS mutant cases more commonly in codon $13(30 \%)$ than in codon $12(17 \%) .^{9}$ These authors demonstrated that KRAS codon 13 MASI is an independent adverse prognostic factor and suggested that it may also influence the response to anti EGFR treatment. ${ }^{9}$ Thus, quantifying mutant allele may be considered when reporting the KRAS mutational status. ${ }^{12}$ However, none of the studies considered whether KRAS MASI occurs evenly in tumour tissue or it has a subclonal distribution. Thus, further investigation is required to confirm and clarify the biological significance and the clinical role of KRAS MASI, also exploiting novel technologies, such as next generation sequencing (NGS) that allow for a quantitative assessment of the mutant allele. ${ }^{13}$

Our molecular laboratory is the largest volume reference centre for community KRAS testing in South Italy. ${ }^{14}$ A large number of sequencing electropherograms derived by our routine practice were reviewed to verify the rate and tissue distribution of KRAS MASI as well as its prognostic clinical relevance. In a subset of samples the sequencing electropherograms semiquantitative review was compared with MASI evaluation by NGS. Moreover, since G13D MASI may have a role as an anti-EGFR therapy predictive marker, ${ }^{9}$ the effect of the G13D copy number on the cetuximab treatment in cell line models was also evaluated.

\section{METHODS \\ KRAS MASI determination}

Since KRAS mutational analysis is part of the routine diagnostic workup of patients with metastatic CRC the need for an ethics committee approval was not necessary for this study, in accordance with medical ethical guidelines of the
Sgariglia R, De Stefano A,

I Clin Pathol 
Azienda Universitaria Policlinico Università degli Studi di Napoli Federico II, and in accordance with general authorisation to process personal data for scientific research purposes from "The Italian Data Protection Authority". To assess KRAS MASI, we quantified the KRAS mutant and wild type allelic peak heights on sequencing electropherograms, by the methods previously described by Soh et al. ${ }^{8}$ To this end, a total of 603 sequencing electropherograms showing a KRAS exon 2 mutant peak in codons 12 or 13 were retrieved from our clinical records. ${ }^{14}$ To limit the confounding effect of background noise, special care was taken to select only electropherograms with an average Phred Quality Score $>20$. On the overall, a total of 437 sequencing electropherograms were included in the study for review. Mutations occurred in $353(80 \%)$ samples in codon 12 (G12D n.=145 (41\%); G12 V n.=111 (31\%); G12C n.=34 (10\%); G12S n.=34 (10\%); G12A n.=22 (6\%); G12R n.=7 (2\%)), while $84(20 \%)$ cases harboured a mutation in codon 13 (G13D n. $=80$ (95\%); G13C n.=4 (5\%)). Differences between type of mutation and MASI occurrence were assessed using Fisher's exact test considering a $\mathrm{p}$ value $\leq 0.05$ as statistically significant.

To verify whether the above described methodology was accurate in MASI detection, 30 cases showing a KRAS MASI on the sequencing electropherograms review and having sufficient (>50 ng) banked DNA were selected to perform KRAS exon 2 mutational analysis by NGS. To this end, extracted DNA was analysed by using the 454 GS-Junior sequencer (Roche Diagnostics, Mannheim, Germany) at the Molecular Pathology facility of the Bellaria Hospital-University of Bologna, as previously described. ${ }^{15}{ }^{16}$ KRAS MASI positive samples were defined as those yielding more mutant reads than wild type ones.

Five primary tumours showing KRAS MASI were further investigated to assess whether this phenomenon had a homogeneous or heterogeneous tissue spatial distribution. To this end, in each case, five different tissue areas were sampled by a laser capture microdissector (Carl Zeiss, Palm Microsystems) (figure 1). Each area of at least $150000 \mu \mathrm{m}^{2}$ was independently analysed for mutational status of codons 12 and 13 of exon 2 of $K R A S$, as previously reported, ${ }^{17}$ assessing on the corresponding sequencing electropherogram KRAS MASI occurrence. ${ }^{14}$
Statistical analysis and MASI clinical significance evaluation

To assess the relevance of KRAS MASI as a prognostic factor, we selected 58 patients, $(n=20$ positive for MASI; $n .=38$ negative for MASI), whose clinical data were available. Univariate analysis for overall survival (OS) was performed using the Kaplan-Meier methods. The OS was calculated as the time between the first visit with the oncologist and the last visit or death from any cause of the patient. All analyses were performed using SPSS IBM software (Milan, Italy) considering a $\mathrm{p}$ value $\leq 0.05$ as statistically significant.

\section{Cell culture, transfections and pharmacological treatments}

To assess whether G13D MASI may influence the in vitro response to anti-EGFR therapy the effect of KRAS MASI on the cetuximab treatment in cell lines models was evaluated. To this end, SW48 colorectal carcinoma cell line was maintained as previously decribed, ${ }^{18}$ and transfected with Lipofectamine 2000 (Life Technologies, Grand Island, New York, USA) using $0.5 \mu \mathrm{g}$ and $2 \mu \mathrm{g}$ of myc-KRAS-G13D and $0.5 \mu \mathrm{g}$ of $m y c-K R A S-\mathrm{G} 12 \mathrm{~V}$ plasmid vectors, ${ }^{19}$ following manufacturer's suggestions. Cells were treated by cetuximab (Erbitux, Merck Serono, Darmstadt, Germany) for $48 \mathrm{~h}$, in triplicate. Cell viability was evaluated by using the CellTiter 96 AQueous One Solution Cell Proliferation Assay (Promega, Madison, Wisconsin, USA) following manufacturer's instructions.

\section{RESULTS}

\section{Frequency and distribution of MASI}

In 56/437 (12.8\%) cases KRAS MASI was observed. Of these, in 40 cases MASI occurred in codon $12((\mathrm{G} 12 \mathrm{D}=19(48 \%)$; $\mathrm{G} 12 \mathrm{~V}=4$ (10\%); G12S=7 (17\%); G12A=6 (14\%); G12C=3 $(7 \%) ; \mathrm{G} 12 \mathrm{R}=1(2 \%))$, whereas 15 cases harboured codon 13 MASI. MASI was more frequently associated with G13D mutation $(\mathrm{p}=0.05)$.

The comparative analysis of KRAS MASI detection by Sanger Sequencing and NGS showed 94\% (28/30) concordance. Only two cases $(6 \%)$ showing KRAS MASI by Sanger Sequencing had discordant result by NGS.
Figure 1 Mutant allele specific imbalance (MASI) spatial tissue distribution. Note discordance in KRAS MASI tissue distribution. G13D KRAS MASI occurred in B1 and B2 sequencing electropherograms, whereas $\mathrm{A} 1, \mathrm{~A} 2$ and $\mathrm{C}$ lacked MASI.

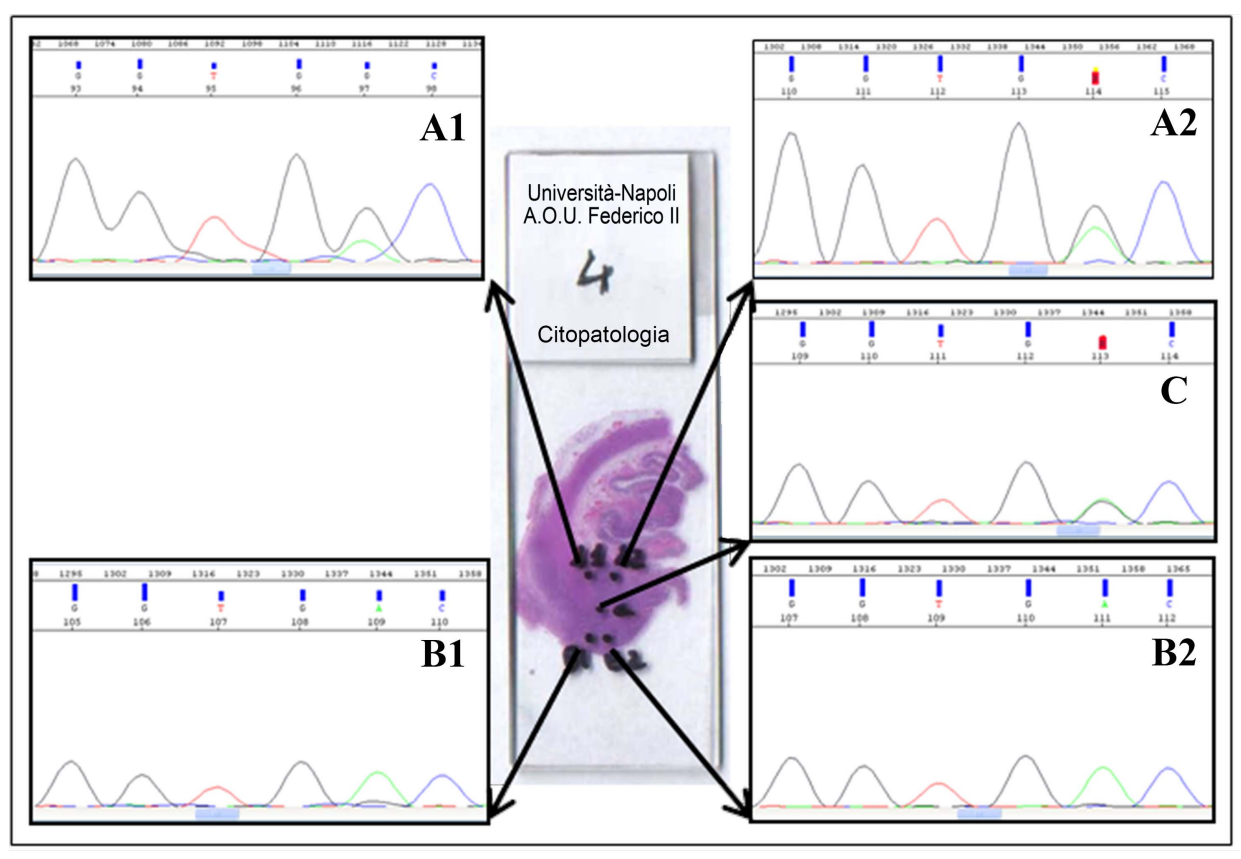


Table 1 Masi Tissue Spatial Distribution

\begin{tabular}{l|lllll}
\hline & 1 & 2 & 3 & 4 & 5 \\
\hline$A 1$ & & & & & \\
A2 & & & & & \\
$B 1$ & & & & & \\
$B 2$ & & & & \\
\hline
\end{tabular}

Tissue spatial distribution of G13D MASI (blue boxes MASI positive; white boxes MASI negative) in different laser microdissected tissue areas from five tumours. Four cases (1-4) analysed showed concurrent MASI positive (blue) and negative (white) tissue areas. Only in one instance (case 5) KRAS MASI was observed in all selected areas.

MASI, mutant allele specific imbalance.

Data relative to the independent assessment of laser microdissected different tissue areas from five single tumours are reported in table 1 . Only in one instance (case 5) KRAS MASI was observed in all selected areas. Conversely, the other four cases analysed showed concurrent MASI positive and negative tissue areas (figure 1).

\section{Evaluation of the clinical significance of MASI}

The Kaplan-Meier curve relative to the comparison in OS between the groups of patients with KRAS MASI $(n=20$; OS: $74.1 ; 95 \%$ CI 15.1 to 133.1$)$ and without ( $n=38$; OS: 172.1 ; $95 \%$ CI 163.0 to 181.3 ) is shown in figure 2. The longer OS in the KRAS MASI negative patient group did not reach statistical significance $(\mathrm{p}=0.08)$.
Measurement of cetuximab sensitivity in G13D cell line harbouring different MASI status

To evaluate the effect of KRAS MASI G13D on cetuximab treatment, SW48 cells were transfected with increasing amount of myc-KRAS-G13D plasmid vector, using as a control, cells were transfected by $0.5 \mu \mathrm{g}$ of myc-KRAS-G12 V plasmid vector. The enforced expression of myc-KRAS-G13D, compared with KRAS G12 V transfected cell lines conferred dose dependent resistance to cetuximab treatment (figure 3).

\section{DISCUSSION}

In this study, in addition to confirm that KRAS MASI is not uncommon, several new findings were presented. Previously, Hartman et $a l^{9}$ found MASI in $19.7 \%$ of KRAS mutated CRCs. This is not dissimilar from our series rate of $12.8 \%$. Also similar to Hartman, KRAS MASI was more frequently observed in association with G13D mutation $(p=0.05)$. Detection of MASI of an oncogene requires, besides the detection of a mutation, the determination of the relative mutant and wild type allele ratio. ${ }^{8}$ Previous studies have consistently measured mutant allelic peak height on the sequencing electropherograms. ${ }^{9} 1012$ Soh et al ${ }^{8}$ showed that this method is as accurate as subcloning to quantify the relative ratio between mutant and wild type allele. Thus, the simple review of diagnostic sequencing electropherograms avoids laborious and time-intensive subcloning, making it possible to identify MASI in clinical practice. ${ }^{9} 10$ Indeed, this approach has been exploited in several studies that investigated the relevance of oncogenic MASI in lung, ${ }^{10} 2021$ pancreatic $^{11}$ and colon ${ }^{9}$ carcinomas for a number of oncogenes including $K_{R A S^{10}} 1120$ and EGFR. ${ }^{21}$ More recently, NGS is replacing Sanger Sequencing to identify clinical actionable mutations in
Figure 2 Kaplan-Meier overall survival curves compared the group of patients with mutant allele specific imbalance (MASI) (green line) against the one without (blue line). The difference between the two groups of patients did not reach the statistical significance $(p=0.08)$.

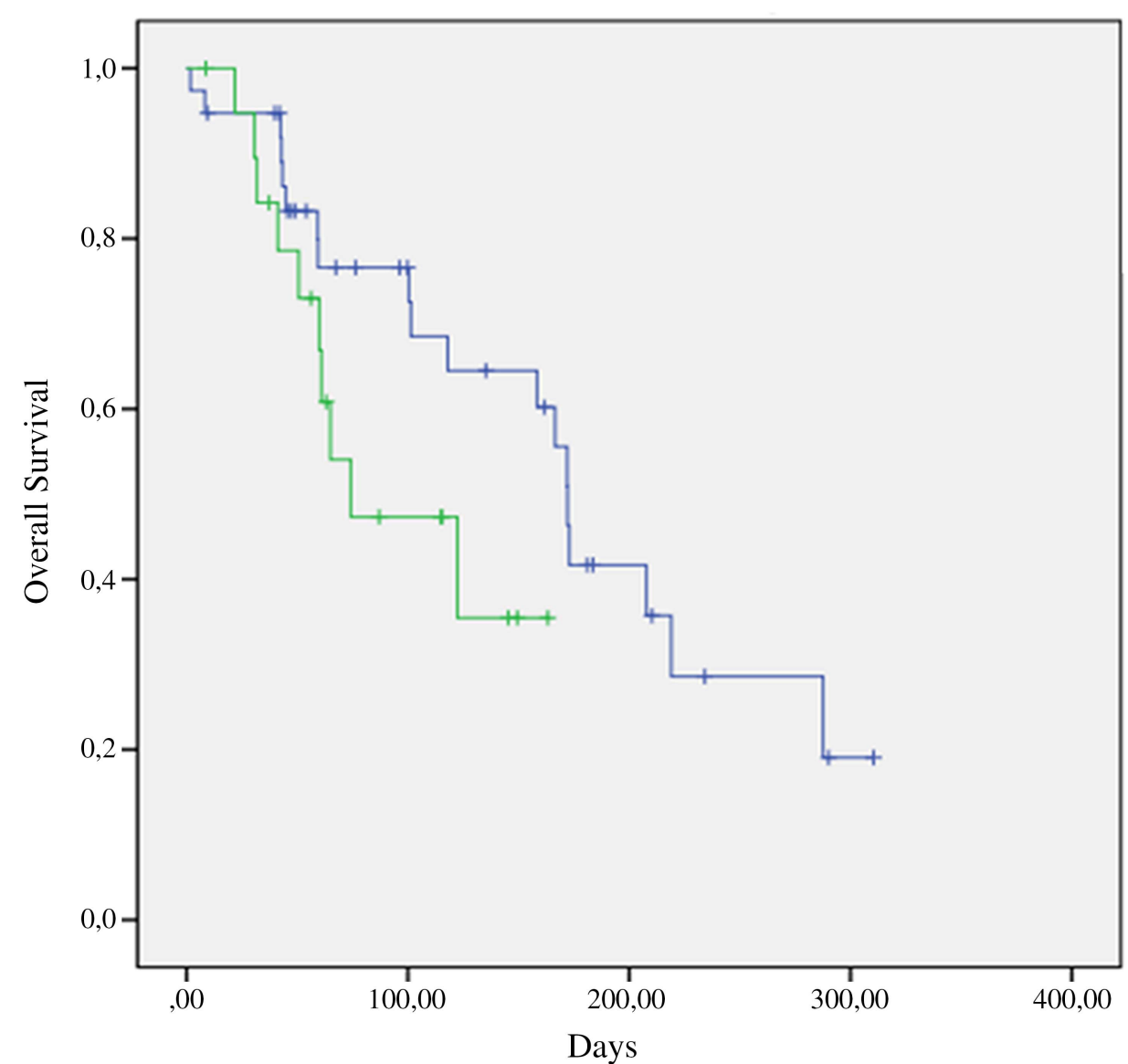




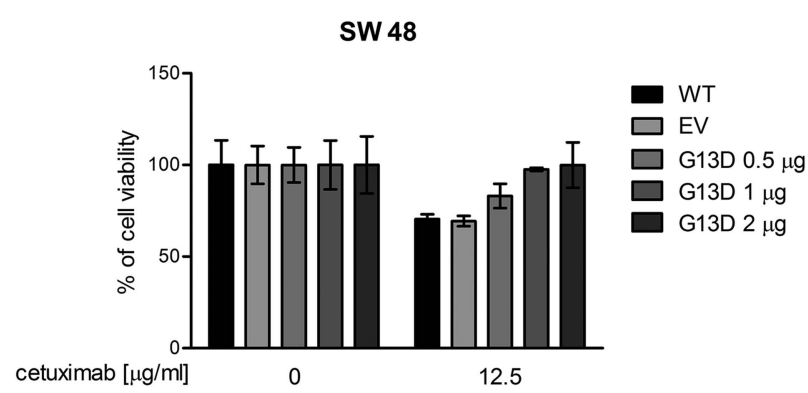

Figure 3 The effect of myc-KRAS-G13D transfection on SW 48 cell line viability. Note that the enforced expression of myc-KRAS-G13D vector increases the percentage of cell viability. Myc-KRAS-G12V transfected cells were used as a control. MASI, mutant allele specific imbalance.

many diagnostic settings. ${ }^{22} 23$ Moreover, NGS allows for a highly accurate quantitative assessment of mutant allele in solid tumours. $^{13}$ To date, NGS has not been employed to investigate MASI. In this study, we showed that in most cases (94\%) the identification of MASI by NGS is concordant with that by direct sequencing electropherograms review. Only in 6\% of cases MASI identified by Sanger sequencing was not confirmed by NGS. Limits of Sanger Sequencing partly depend on the specific mutation detection and on the different intensities of fluorescent emissions related to different nucleotides.

In previous studies, KRAS MASI was exclusively evaluated on DNA extracted from a whole paraffin section of neoplastic tissue, without taking into account different areas of the same tumour. ${ }^{10} 11{ }^{20}$ In this study, we investigated whether MASI has a homogeneous or a heterogeneous tissue distribution. To avoid dilution of mutant allele into benign tissue-derived DNA, neoplastic-rich areas were sampled by laser capture microdissection. Using this approach we demonstrated that KRAS MASI has an uneven tissue distribution (figure 1). Thus, while KRAS mutation is an early event in colorectal tumours, ${ }^{7}$ it can be argued that KRAS MASI may be related to clonal selection during tumour progression. A discordant KRAS mutational status between primary tumour and the corresponding metastasis is not exceptional, when using a less sensitive technique. ${ }^{7}$ From a technical standpoint it can be argued that the occurrence of MASI during tumour progression may lead to a different number of mutant allele between the primary and the metastatic sites.

Hartman et $a l^{9}$ showed that KRAS MASI positive patients with CRC have a worse prognosis. Similar evidence was reported for lung ${ }^{10}$ and pancreatic ${ }^{11}$ neoplasms. Our data showed longer OS of KRAS MASI negative patients. However, this finding is not statistically significant $(p=0.08)$. Our retrospective study analysed a large number of routine sequencing electropherograms, but it was not specifically designed to establish the impact of KRAS MASI on the OS in a large cohort of patients with CRC.

The response of patients with CRC harbouring KRAS G13D mutation to anti-EGFR is currently being investigated. Hartman et al suggested that ongoing clinical trials on anti-EGFR therapy in KRAS p.G13D-mutated CRC should take into account KRAS MASI status. We evaluated in vitro the effect of KRAS MASI on cetuximab treatment. Our results showed that the enforced expression of KRAS G13D mutation, using as a control G12 V mutation, was able to confer resistance to treatment with cetuximab in a dose-dependent manner (figure 3), strongly supporting the idea that allelic imbalance could be responsible of the observed resistance. Interestingly, Valtorta et $a l^{24}$ using preclinical models and patients' samples recently reported that the emergence of KRAS amplification is associated with acquired resistance to the EGFR inhibitors.

In conclusion, this study confirmed that KRAS MASI is significant in CRC. Among codon 12 and 13 KRAS mutations, MASI is specifically associated with G13D mutation, and this may play a role in predicting the response to EGFR inhibitors. KRAS MASI may be a late event in tumour progression, as suggested by its heterogeneous spatial distribution. Future studies designed to assess KRAS MASI in larger cohorts of patients, in prognostic and predictive terms, may exploit NGS as an investigational tool.

\section{Take home messages}

- KRAS mutant allele-specific imbalance (MASI) can be identified when the mutant allele peak is higher than the wild type one on the sequencing electropherogram.

- KRAS MASI is not infrequent and it has a heterogeneous intratumoral distribution. It is more commonly associated with the G13D mutation.

- The in vitro model showed that KRAS MASI represents a mechanism of resistance to cetuximab treatment.

\section{Handling editor Runjan Chetty}

Contributors UM and GTr: conceived and designed the experiments; UM, RS and DdB: performed the experiments; UM, GTr, ADS, CC, EV, PP, CB, RS and GTa: analysed the data; UM, EV, CB, PP, GTa and GTr; contributed to the writing of the manuscript.

\section{Competing interests None.}

Patient consent Obtained.

Ethics approval Institutional Review Board, Carlo Romano.

Provenance and peer review Not commissioned; externally peer reviewed.

\section{REFERENCES}

1 Parkin DM, Bray F, Ferlay J, et al. Global cancer statistics, 2002. CA Cancer J Clin 2005;55:74-108

2 Ciardiello F, Tortora G. EGFR antagonists in cancer treatment. N Eng/ J Med 2008;358:1160-74.

3 Amado RG, Wolf $M$, Peeters $M$, et al. Wild-type KRAS is required for panitumumab efficacy in patients with metastatic colorectal cancer. J Clin Oncol 2008:26:1626-34.

4 Peeters M, Price TJ, Cervantes A, et al. Randomized phase III study of panitumumab with fluorouracil, leucovorin, and irinotecan (FOLFIRI) compared with FOLFIRI alone as second-line treatment in patients with metastatic colorectal cancer. J Clin Oncol 2010;28:4706-13.

5 Van Cutsem E, Kohne CH, Lang I, et al. Cetuximab plus irinotecan, fluorouracil, and leucovorin as first-line treatment for metastatic colorectal cancer: updated analysis of overall survival according to tumor KRAS and BRAF mutation status. I Clin Oncol 2011;29:2011-19.

6 Van Cutsem E, Kohne $\mathrm{CH}$, Hitre $\mathrm{E}$, et al. Cetuximab and chemotherapy as initial treatment for metastatic colorectal cancer. N Engl J Med 2009;360:1408-17.

7 Malapelle U, Carlomagno C, de Luca C, et al. KRAS testing in metastatic colorectal carcinoma: challenges, controversies, breakthroughs and beyond. J Clin Pathol 2014;67:1-9.

8 Soh J, Okumura N, Lockwood WW, et al. Oncogene mutations, copy number gains and mutant allele specific imbalance (MASI) frequently occur together in tumor cells. PLOS ONE 2009;4:e7464.

9 Hartman DJ, Davison JM, Foxwell TJ, et al. Mutant allele-specific imbalance modulates prognostic impact of KRAS mutations in colorectal adenocarcinoma and is associated with worse overall survival. Int J Cancer 2012;131:1810-17.

10 Chiosea $\mathrm{SI}$, Sherer $\mathrm{CK}$, Jelic T, et al. KRAS mutant allele-specific imbalance in lung adenocarcinoma. Mod Pathol 2011;24:1571-7. 
11 Krasinskas AM, Moser AJ, Saka B, et al. KRAS mutant allele-specific imbalance is associated with worse prognosis in pancreatic cancer and progression to undifferentiated carcinoma of the pancreas. Mod Pathol 2013;26:1346-54.

12 Hartman DJ, Chiosea SI. Colorectal carcinomas, KRAS p.G13D mutant allele-specific imbalance, and anti-epidermal growth factor receptor therapy. Cancer 2013;119:4366.

13 Portier BP, Kanagal-Shamanna R, Luthra R, et al. Quantitative assessment of mutant allele burden in solid tumors by semiconductor-based next-generation sequencing. Am J Clin Pathol 2014;141:559-72.

14 Malapelle U, Bellevicine C, Salatiello $M$, et al. Sanger sequencing in routine KRAS testing: a review of 1720 cases from a pathologist's perspective. J Clin Pathol 2012:65:940-4.

15 de Biase $D$, Visani $M$, Baccarini $P$, et al. Next generation sequencing improves the accuracy of KRAS mutation analysis in endoscopic ultrasound fine needle aspiration pancreatic lesions. PLOS ONE 2014;9:e87651.

16 de Biase D, Visani M, Malapelle U, et al. Next-Generation Sequencing of Lung Cancer EGFR Exons 18-21 Allows Effective Molecular Diagnosis of Small Routine Samples (Cytology and Biopsy). PLoS ONE 2013;8:e83607.

17 Malapelle U, de Rosa N, Rocco D, et al. EGFR and KRAS mutations detection on lung cancer liquid-based cytology: a pilot study. J Clin Pathol 2012;65:87-91.
18 Pallante $\mathrm{P}$, Malapelle $\mathrm{U}$, Berlingieri MT, et al. UbcH10 overexpression in human lung carcinomas and its correlation with EGFR and p53 mutational status. Eur J Cancer 2013:49:1117-26.

19 Kumar SS, Price TJ, Mohyieldin 0, et al. KRAS G13D Mutation and Sensitivity to Cetuximab or Panitumumab in a Colorectal Cancer Cell Line Model. Gastrointest Cancer Res 2014;7:23-6.

20 Villaruz LC, Socinski MA, Cunningham DE, et al. The prognostic and predictive value of KRAS oncogene substitutions in lung adenocarcinoma. Cancer 2013; 119:2268-74.

21 Oakley III GJ, Chiosea SI. Higher dosage of the epidermal growth factor receptor mutant allele in lung adenocarcinoma correlates with younger age, stage IV at presentation, and poorer survival. J Thorac Oncol 2011;6:1407-12.

22 Sikkema-Raddatz B, Johansson LF, de Boer EN, et al. Targeted next-generation sequencing can replace Sanger sequencing in clinical diagnostics. Hum Mutat 2013;34:1035-42.

23 Malapelle U, Vigliar E, Sgariglia R, et al. Ion Torrent next-generation sequencing for routine identification of clinically relevant mutations in colorectal cancer patients. J Clin Pathol 2015;68:64-8.

24 Valtorta E, Misale S, Sartore-Bianchi A, et al. KRAS gene amplification in colorectal cancer and impact on response to EGFR-targeted therapy. Int J Cancer 2013;133:1259-65. 